\title{
Los Herbicidas, Una Alternativa para el Control de la Maleza Spergula arvensis en el Cultivo de Papa en Bolivia
}

\author{
Pablo Mamani ${ }^{1}$,René Pereira ${ }^{1}$, André Devaux ${ }^{2}$
}

\section{Resumen}

En dos campañas consecutivas (1993-94 y 1994-95) en la Estación Experimental Toralapa se buscó determinar "La época más apropiada de aplicación del herbicida Sencor (Metribuzin)" y seleccionar "Nuevas alternativas de herbicidas" ambos para el control de la maleza Spergula arvensis en el cultivo de papa. Para el primer caso, en la primera campaña se utilizaron los siguientes tratamientos: Aplicación pre-emergente, aplicación luego del aporque, aplicación a la floración y aplicación pre-emergente más aplicación a la floración, frente a un testigo sin aplicación. En base a los resultados en la campaña siguiente se probaron los siguientes tratamientos: Aplicación pre-emergente, aplicación luego del aporque y aplicación pre-emergente más $50 \%$ luego del aporque, frente a un testigo sin aplicación. Para el segundo caso, en la primera campaña se utilizaron los siguientes herbicidas: Sencor (Metribuzin) Cention (Diuron), Herbadox (Phendimetalin) y Basagran (Bentazon), todos en dos dosis, una recomendada por la fábrica y la otra igual al $75 \%$ de dicha dosis. En la campaña siguiente se verificó el efecto de los mismos herbicidas y se incluyeron los siguientes: Gesagart (Prometrina); lloxan (Diclorofop metil) y Triflurex (Trifluralina). En la primera campaña el aporque se efectuó en forma manual y en la segunda campaña en forma mecanizada.

Los resultados encontrados permiten señalar que el herbicida Sencor independientemente de la época de su aplicación controla eficientemente esta maleza, pero la aplicación pre-emergente permitió además un incremento substancial en el rendimiento respecto a la aplicación pos-emergente en general.

\footnotetext{
${ }^{1}$ Fundación PROINPA, Casilla 4285, Cochabamba, Bolivia. Correo electrónico: pmamani @ proinpa.org

2 Centro Internacional de la Papa (CIP), Lima, Perú.
} 
Si bien el aporque manual controló mejor la maleza respecto al aporque mecánico, su costo por mano de obra y su poca efectividad en condiciones extensivas deben ser consideradas. Se debería dar más atención al deshierbe mecánico.

En cuanto a las nuevas opciones de herbicidas se puede señalar que Sencor, Herbadox y Basagran, además de controlar la maleza tuvieron un efecto positivo en el rendimiento total. Contrariamente el herbicida Cention aunque controló efectivamente a la maleza, tuvo un efecto negativo en el rendimiento del cultivo. En ambas campañas los mayores beneficios económicos se obtuvieron con los productos Sencor, Herbadox y Basagran, pudiendo recomendarse a estos dos últimos como alternativas para el control de $S$. arvensis en el cultivo de la papa.

Palabras claves adicionales: Spergula arvensis, herbicidas, malezas, control químico, Metribuzin, Diuron, Phendimetalin, Prometrina, Bentazon, Diclofop metil, Trifluralina.

\section{Herbicides, An Alternative for the Control of Spergula arvensis Need in Potato Cultivation in Bolivia}

\section{Summary}

Research for determining the "Most appropriate time to apply the Sencor (Metribuzin) herbicide" and to select "new herbicide alternatives" was conducted in two consecutive growing seasons (1993-94 and 1994-95) at Toralapa Experimental Station to control Spergula arvensis in potato crops.

To achieve the first objective, the following treatments were performed during the first season: pre-emergence application with after-hilling application and application at blooming, and pre-emergence application with application at blooming against control without applications. Based on the results of the first season, in the following one the following treatments were performed: pre-emergence application with after-hilling application and pre-emergence application with $50 \%$ after-hilling application, and compared to a control without application. 
To achieve the second objective, during the first season 4 different herbicides were used: Sencor (Metribuzin), Cention (Diuron), Herbadox (Phendimetalin) and Basagran (Bentazon) in two different doses, the one suggested by the manufacturers and another equal to the $75 \%$ of it. In the second season, the same herbicides were used together with three new herbicides: Gesagart (Prometrina), Iloxan (Diclorofop metil) y Triflurex (Trifluralina). In the first season, hilling was done manually and in the second was done mechanically.

The results confirmed that Sencor achieved an efficient control, independently from the time of the application but, with a preemergence application, yield was improved substantially compared to all the post-emergence applications.

Even though the manual hilling controlled the weed in a better way than the mechanical hilling, this technique should be evaluated regarding its cost and little effectiveness in extensive conditions. More attention should be given to improving mechanical weeding.

Regarding the new herbicides, we can say that Sencor, Herbadox, and Basagran, besides controlling the weed, had a positive effect on yield. The Cention herbicide had a negative effect on yield although it had a good effect on controlling the weed. In both seasons, greater economic benefits were obtained with Sencor, Herbadox, and Basagran being the last two recommended as an alternative for controlling S. arvensis in potato crops.

Additional Index words: Spergula arvensis, herbicides, weeds, Chemical control, Metribuzin, Diuron, Phendimetalin, Prometrina, Bentazon, Diclofop metil, Trifluralina.

\section{Introducción}

Las malezas no constituyen un factor importante en la producción de papa en la zona andina de Bolivia, debido al sistema tradicional de producción y al efecto competidor del cultivo. Sin embargo, en los últimos años, en las zonas de altura de las provincias Carrasco, 
Tiraque, Arani y Chapare del departamento de Cochabamba, se ha magnificado el problema de la maleza $S$. arvensis L, no sólo en papa sino también en cereales. Aunque no se reportó la magnitud de su efecto negativo en el cultivo de papa, se sabe de su efecto en cereales menores (trigo y cebada) y en haba (Terrazas et al, 1994), que constituyen los principales cultivos en el sistema de rotación de muchos agricultores.

S. arvensis es una especie anual de hoja angosta, dicotiledónea, pertenece a la familia Caryophyllaceae. Según Terrazas y Germain (1994), está adaptada a suelos livianos y ácidos (pH entre 4 y 6) con buena humedad y fertilidad. Su crecimiento puede alcanzar hasta 75 $\mathrm{cm}$ de altura, produce entre 1,000 y 30,000 frutos por planta, hasta 45 semillas por fruto, cerca del millón de semillas por planta. Su germinación es mucho mayor con un desterronado fino en la preparación del suelo. Por otra parte, esta maleza posee buenas cualidades forrajeras y es apetecida por el ganado.

La alta capacidad de producción de semilla de esta maleza, su fácil dispersión y su gran capacidad de persistencia (por su largo período de viabilidad y por su germinación escalonada), hacen de esta maleza un enemigo en potencia importante para el cultivo de la papa.

Como lo muestran Terrazas y Germain (1994), Spergula arvensis también se constituye como maleza en los cultivos de rotación más tradicionales en las zonas de altura de Cochabamba, como lo son la cebada y el haba. Referente al control mecánico, la experiencia en la Estación Experimental Toralapa (consulta personal) muestra que las labores de aradura con el propósito de exponer las semillas de esta maleza a la acción desecante del sol tiene poco efecto, atribuible posiblemente al largo período de viabilidad y a la germinación escalonada de esta semilla. Por otra parte, un aporque oportuno y bien ejecutado en forma manual controla efectivamente a esta maleza. Sus limitantes en condiciones extensivas son su costo por mano de obra, los daños que se ocasionan a los órganos subterráneos de la planta y la poca efectividad en el control de las malezas que se encuentran sobre el surco (entre plantas del cultivo).

Si bien las malezas deben manejarse en base a programas integrales que utilicen en forma óptima todos los medios de prevención, erradicación y control disponibles, en el caso particular de la maleza S. arvensis cuyo daño al cultivo de papa es doblemente importante 
por ser además de competidora, hospedante de $N$. aberrans (nematodo importante en el cultivo de la papa), su control constituye la práctica más viable y dentro de ella el control químico con todas sus posibles limitaciones ambientales, debe ser considerado como una alternativa a corto plazo que pueda ser aplicado en sistemas de producción intensivos con recomendaciones específicas para su manejo eficiente.

Por lo expuesto, el objetivo del presente trabajo de investigación fue determinar la época de aplicación del herbicida Sencor (Metribuzin) para el control de $S$. arvensis y seleccionar herbicidas pre y posemergentes con amplio poder de control de dicha maleza en el cultivo de la papa.

\section{Materiales y Métodos}

Los resultados corresponden a ensayos conducidos en dos campañas consecutivas (1993/94 y 1994/95), descritos en la Tabla 1. Estos ensayos se realizaron en la Estación Experimental Toralapa, provincia Tiraque del departamento de Cochabamba. Geográficamente ubicada a $17^{\circ} 31^{\prime}$ de latitud sur y $65^{\circ} 40^{\prime}$ de longitud oeste, a una altura de $3430 \mathrm{msnm}$. La temperatura media anual es de $11^{\circ} \mathrm{C}$ y la precipitación media anual de $530 \mathrm{~mm}$. Sus suelos son de origen aluvial, de textura franca limosa y de $\mathrm{pH}$ ácido (5 a 6).

En el Ensayo 1 se utilizó el herbicida Sencor por ser el más comercial. Los herbicidas utilizados en el Ensayo 2 de la campaña 1993-94 tienen antecedentes en Brasil para el control de S. arvensis (Kurt Von Hertwing, 1983). En el Ensayo 2 de la Campaña 1994-95 se incluyeron otros herbicidas, manteniendo aquellos de la campaña anterior para su verificación.

A continuación se detallan las dosis utilizadas en los ensayos, basadas en recomendaciones de la fábrica para papa y/o cultivos afines: Sencor $=1.5 \mathrm{It} / \mathrm{ha}$, Basagran $=1.5 \mathrm{l} / \mathrm{ha}$, Herbadox $=3 \mathrm{l} / \mathrm{ha} \mathrm{y}$ Cention $=1.5 \mathrm{~kg} / \mathrm{ha}$, Gesagart $=1.5 \mathrm{l} / \mathrm{ha}$, Iloxan $=3 \mathrm{l} / \mathrm{ha}$ y Triflurex = 1.2 l/ha. La dosis mínima (Dm) en el Ensayo 2 de la Campaña 199394 fue igual al 75\% de la dosis recomendada (DR) de cada producto. 
Tabla 1. Descripción de los ensayos de control químico de la maleza S. arvensis en el cultivo de papa, conducidos en dos campañas en la Estación Experimental Toralapa.

\begin{tabular}{|c|c|c|c|c|}
\hline \multirow{3}{*}{ Diseño } & \multicolumn{2}{|c|}{ CAMPAÑA 1993 -1994 } & \multicolumn{2}{|c|}{ CAMPAÑA 1994-1995 } \\
\hline & Ensayo 1 & Ensayo 2 & Ensayo 1 & Ensayo 2 \\
\hline & BCA con 4 repetic. & $\begin{array}{l}\text { BCA con arreglo factorial (4 } \\
\text { herb. y } 2 \text { dosis) con } 4 \text { rep. }\end{array}$ & BCA con 4 repeticiones & BCA con 4 repeticiones \\
\hline Factor & $\begin{array}{l}\text { 1) Época de aplicación de } \\
\text { Sencor }\end{array}$ & $\begin{array}{l}\text { 1) Herbicida } \\
\text { 2) Dosis }\end{array}$ & $\begin{array}{l}\text { 1) Época de aplicación de } \\
\text { Sencor }\end{array}$ & 1) Herbicida \\
\hline Tratam. & $\begin{array}{l}\text { 1) Sin aplicación (testigo) } \\
\text { 2) Aplic. pre-emergente } \\
\text { 3) Aplic. luego del aporque } \\
\text { 4) Aplic. a la floración } \\
\text { 5) Aplic. preemergente y a } \\
\text { la floración }\end{array}$ & $\begin{array}{l}\text { 1) Sencor (Metribuzin) x DR } \\
\text { 2) Sencor (Metribuzin) x Dm } \\
\text { 3) Basagran (Bentazon) } \times \mathrm{DR} \\
\text { 4) Basagran (Bentazon) } \times \mathrm{Dm} \\
\text { 5) Cention } 80 \text { (Diuron) } \times \mathrm{DR} \\
\text { 6) Cention } 80 \text { (Diuron) } \times \mathrm{Dm} \\
\text { 7) Herbadox } 33 \mathrm{E} \\
\text { (Pendimetalin) } \times \mathrm{DR} \\
\text { 8) Herbadox } 33 \mathrm{E} \\
\text { (Pendimetalin) } \times \mathrm{Dm}\end{array}$ & $\begin{array}{l}\text { 1) Sin aplicación (testigo) } \\
\text { 2) Aplic. preemergente } \\
\text { 3) Aplic. luego del aporque } \\
\text { 4) Aplic. preemergente más } \\
50 \% \text { luego del aporque }\end{array}$ & $\begin{array}{l}\text { PRE-EMERGENTES } \\
\text { 1) Sencor (Metribuzin) } \\
\text { 2) Cention } 80 \text { (Diuron) } \\
\text { 3) Herbadox } 33 E \\
\text { (Pendimetalin) }\end{array}$ \\
\hline
\end{tabular}

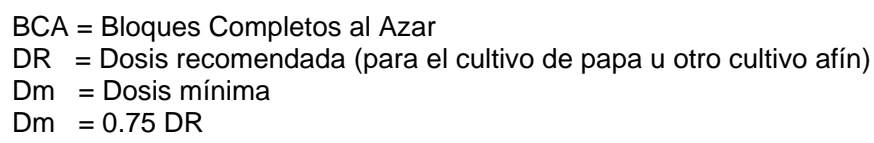

El tamaño de las unidades experimentales fue de $6 \mathrm{~m}$ de largo por 4.9 $\mathrm{m}$ de ancho (7 surcos por unidad experimental separados a $0.7 \mathrm{~m}$ ).

La aplicación de los herbicidas se realizó con una mochila manual cuya boquilla de aspersión permitía una distribución en "abanico" para cubrir mejor el área por aplicar. Las aplicaciones pos-emergentes se realizaron en forma dirigida a la maleza, mojando en los entresurcos y evitando mojar el cultivo.

En todos los ensayos se utilizó la variedad Waych'a, por ser la más comercial en los mercados bolivianos. Las variables evaluadas en la Campaña 1993-94 fueron: número de plantas de maleza $/ \mathrm{m}^{2}$ a pocos días de su emergencia y a inicios de su fase reproductiva (floración), 
materia seca de la maleza, grado de control de la maleza mediante el uso de una escala (1= Control absoluto, 2=Buen control, 3=Regular control, 4=Mal control y $5=$ Sin control), emergencia del cultivo, cobertura foliar del cultivo, rendimiento del cultivo (total y por categorías). En la Campaña 1994-95 sólo se evaluó el rendimiento del cultivo (total y por categorías).

El aporque es una práctica cultural que se realiza cuando la planta tiene una altura entre 15 a $20 \mathrm{~cm}$ y que consiste en aflojar el suelo del entresurco para mejorar su aireación y cubrir la base de las plantas con el suelo aflojado y así estimular a una mejor tuberización. Esta labor para la primera campaña se efectuó en forma manual utilizando un azadón y para la segunda campaña se utilizó tracción mecánica.

Para el análisis económico se procedió con la metodología de "Presupuestos Parciales" descrita por Horton (1982).

\section{Resultados}

La falta de lluvias al inicio de la Campaña 1993-94 no favoreció el desarrollo normal de la maleza y posiblemente la efectividad de los herbicidas aplicados en forma pre-emergente, pero a medida que las lluvias se fueron regularizando se observó una acentuada población de malezas en las parcelas sin aplicación de herbicida del ensayo 1 , aunque no en la magnitud de años anteriores. La emergencia del cultivo también fue retrasada por falta de una humedad inicial favorable. En la Campaña 1994-95 se observó una mayor población de la maleza, favorecida por una mayor humedad en el suelo antes y luego de la siembra.

En la Campaña 1993-94, con el aporque manual se eliminó totalmente la maleza, pero luego de esta práctica emergieron nuevas plántulas de esta maleza, provenientes de semillas a la espera de condiciones favorables. En el Ensayo 1, el número de malezas presentes antes del aporque en las parcelas sin aplicación de herbicida (testigo) fue de $11,000 \mathrm{pl} / \mathrm{m}^{2}$ (con $0.008 \mathrm{~g}$ de materia seca/pl) y luego del aporque (a la floración de la maleza) fue de 105 $\mathrm{pl} / \mathrm{m}^{2}$ (con $0.15 \mathrm{~g}$ de materia seca/pl).

En el Ensayo 2 de esta misma campaña, los cuatro herbicidas aplicados en dos dosificaciones controlaron efectivamente a la maleza $S$. arvensis hasta el aporque, a partir de este momento se observó que las semillas de esta maleza germinaron nuevamente en porcentajes mínimos que no lograron competir en forma significativa con el cultivo. 
A la maduración del cultivo, la escala utilizada permitió determinar que el herbicida Cention logró un buen control de la maleza y los herbicidas Sencor, Herbadox y Basagran un control regular, lo que no repercutió en el rendimiento del cultivo, porque éste se encontraba ya en plena maduración.

En ambos ensayos de la Campaña 1993-94, no se encontró un efecto directo significativo de los tratamientos con herbicida en la emergencia del cultivo, pero sí en la cobertura foliar, la cual en el caso del Ensayo 1, se incrementó con la aplicación del herbicida Sencor tanto en forma pre-emergente como con su aplicación a una semana luego del aporque y con ambas aplicaciones. La aplicación al momento de la floración parece no afectar la cobertura foliar, aunque se observó un ligero incremento a finales del cultivo en relación al testigo sin aplicación.

En la Tabla 2 se muestra el Análisis de Varianza para el rendimiento total del cultivo en los cuatro ensayos. En todos se observa un efecto altamente significativo de los herbicidas, cuyas comparaciones de medias se presentan en la Figura 1. En el caso del Ensayo 2 de la Campaña 1993-94, se aprecia también el efecto significativo del factor dosis, atribuible al efecto negativo de la dosis recomendada (DR) del herbicida Cention.

Tabla 2. Análisis de Varianza del rendimiento total de ensayos de control químico de la maleza S. arvensis en el cultivo de papa, conducidos en dos campañas en la Estación Experimental Toralapa.

\begin{tabular}{|c|c|c|c|c|c|c|c|c|c|c|c|}
\hline \multicolumn{6}{|c|}{ Campaña 1993-1994 } & \multicolumn{6}{|c|}{ Campaña 1994-1995 } \\
\hline \multicolumn{3}{|c|}{ Ensayo 1} & \multicolumn{3}{|c|}{ Ensayo 2} & \multicolumn{3}{|c|}{ Ensayo 1} & \multicolumn{3}{|c|}{ Ensayo 2} \\
\hline FV & GL & $\mathrm{CM}$ & FV & CM & GL & FV & GL & CM & $\mathrm{FV}$ & GL & CM \\
\hline $\begin{array}{l}\text { Rep. } \\
\text { Sencor }\end{array}$ & $\begin{array}{l}3 \\
4\end{array}$ & $\begin{array}{c}5.01 \\
106.64^{\star \star}\end{array}$ & Rep. & 3 & 20.66 & Rep. & 3 & 1.12 & Rep. & 3 & $16.69^{\star \star}$ \\
\hline \multirow[t]{4}{*}{ Error } & 12 & 9.03 & Herb. $(\mathrm{H})$ & 3 & $212.01^{* *}$ & Sencor & 3 & $140.35^{\star *}$ & Herb. & 6 & $26.10^{\star \star}$ \\
\hline & & & Dosis(D) & 1 & $97.39 * \star$ & Error & 9 & 6.03 & Error & 18 & 3.3 \\
\hline & & & $H \times D$ & 3 & 30.77 & & & & & & \\
\hline & & & Error & 21 & 13.44 & & & & & & \\
\hline \multicolumn{3}{|c|}{ CV: $16.5 \%$} & \multicolumn{3}{|l|}{ CV: $15.0 \%$} & \multicolumn{3}{|c|}{ CV: $21.9 \%$} & \multicolumn{3}{|c|}{ CV: $19.7 \%$} \\
\hline
\end{tabular}

** Significativo al $1 \%$ de probabilidad estadística. 
La Figura 1A correspondiente al Ensayo 1 de la Campaña 1993-94, muestra claramente que las aplicaciones pos-emergentes del herbicida Sencor, son contraproducentes en el rendimiento total del cultivo de papa. La aplicación pre-emergente de este herbicida, tiene un efecto ligeramente superior, aunque no significativo en relación al testigo, pero marcadamente superior al efecto de la aplicación posemergente en general. El poco efecto de la aplicación pre-emergente sobre el testigo se debió a que el aporque manual logró controlar favorablemente a las malezas.

El Ensayo 1 de la Campaña 1994-95 (Figura 1B), confirma la importancia de la aplicación pre-emergente del herbicida Sencor para el control de la maleza S. arvensis en el cultivo de papa. Durante esta campaña el efecto de la aplicación pre-emergente permitió un incremento de rendimiento del $204 \%$ respecto al testigo. Este marcado efecto en relación al testigo muestra que el ahorque mecanizado realizado en esta campaña, no logró un adecuado control de malezas cuando éstas se encuentran sobre el surco. También se puede señalar que es innecesaria una aplicación luego del aporque (pos-emergente) cuando se realiza una aplicación preemergente. Por otra parte resultó poco efectiva la aplicación de este herbicida luego del aporque (pos-emergente), debido a que las malezas se desarrollaron efectivamente antes del aporque aprovechando la humedad del suelo y la poca cobertura por el cultivo. El efecto residual de este herbicida es de 1 a 2 meses, persistiendo normalmente en todo el ciclo del cultivo.

En la Tabla 3 se muestra el análisis económico de los tratamientos no dominados en los ensayos 1 de ambas campañas. La diferencia en el Costo Variable Total entre los tratamientos se debe al precio del producto (Sencor) y a la mano de obra por la aplicación de dicho producto. La Tasa de Retorno Marginal y el Beneficio Neto de ambas campañas muestran la bondad económica de aplicar el herbicida Sencor en forma pre-emergente. Este efecto fue más marcado en la Campaña 1994-95, donde el aporque mecanizado no fue suficiente para controlar a la maleza, por los argumentos mencionados. 
Tabla 3. Análisis marginal de los tratamientos no dominados de los ensayos "Época de aplicación del herbicida Sencor" (ensayos 1).

\section{Campaña 1993-94}

\begin{tabular}{|l|l|c|c|c|c|c|}
\hline No. & Tratamiento & $\begin{array}{c}\text { Beneficio } \\
\text { Neto } \\
(\$ \cup S / h a)\end{array}$ & $\begin{array}{c}\text { Total costos } \\
\text { variables } \\
(\$ \cup S / h a)\end{array}$ & $\begin{array}{c}\text { Beneficio neto } \\
\text { marginal (\$US/ha) })\end{array}$ & $\begin{array}{c}\text { Costo variable } \\
\text { marginal (\$US/ha) })\end{array}$ & $\begin{array}{c}\text { Tasa de } \\
\text { retorno } \\
\text { marginal (\%) }\end{array}$ \\
\hline 2 & $\begin{array}{l}\text { Aplicación Pre- } \\
\text { emergente }\end{array}$ & 2,643 & 41 & 181 & 41 & 441 \\
1 & Sin aplicación & 2,462 & 0 & 0 & 0 & \\
\hline
\end{tabular}

\section{Campaña 1994-95}

\begin{tabular}{|l|l|c|c|c|c|c|}
\hline No. & Tratamiento & $\begin{array}{l}\text { Beneficio } \\
\text { Neto } \\
(\$ \cup S / h a)\end{array}$ & $\begin{array}{c}\text { Total costos } \\
\text { variables } \\
(\$ \cup S / h a)\end{array}$ & $\begin{array}{c}\text { Beneficio neto } \\
\text { marginal (\$US/ha) }\end{array}$ & $\begin{array}{c}\text { Costo variable } \\
\text { marginal }(\$ \cup S / h a)\end{array}$ & $\begin{array}{c}\text { Tasa de } \\
\text { retorno } \\
\text { marginal }\end{array}$ \\
\hline 2 & $\begin{array}{l}\text { Aplicación Pre- } \\
\text { emergente }\end{array}$ & 1,996 & 41 & 1382 & 41 & 3,370 \\
1 & Sin aplicación & 614 & 0 & 0 & 0 & \\
\hline
\end{tabular}

De acuerdo a la Figura $1 C$ en la Campaña 1993-94, las dosis mínimas de los herbicidas Sencor, Basagran y Herbadox, tuvieron un efecto similar a las dosis recomendadas sobre el rendimiento total. Cention tuvo un efecto negativo en el rendimiento, principalmente con la dosis recomendada, debido posiblemente a que este producto normalmente es recomendado para cultivos cuyos sistemas radiculares se desarrollan en su mayor parte en capas profundas. Por otra parte García y Quintanilla (1991), señalan que este producto a dosis no selectivas puede presentar una acción residual por más de un año. 

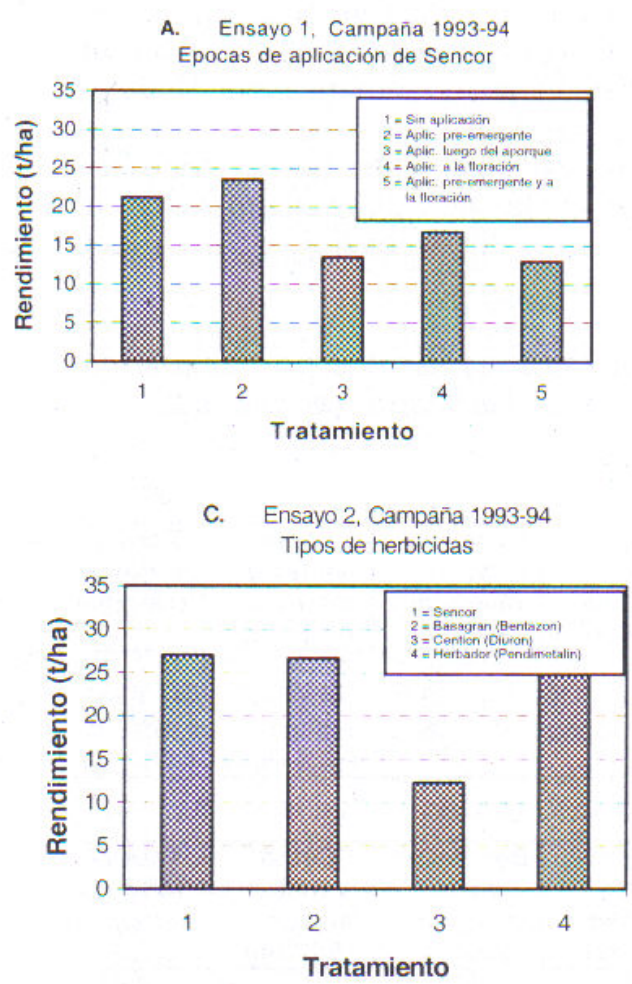
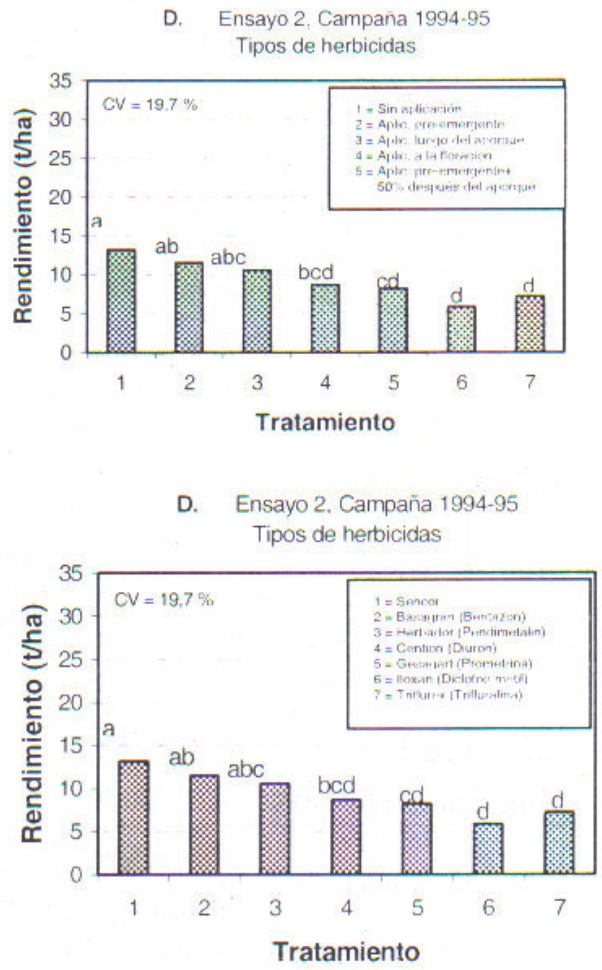

Figura 1. Rendimiento de papa en dos campañas por efecto de la aplicación de herbicidas: $A$ y $B$ ) épocas de aplicación de Sencor (Metribuzin); C y D) tipos de herbicidas.

Continuando en la búsqueda de nuevas alternativas de herbicidas para el control de malezas en el cultivo de la papa, en la Campaña 1994-95 (Ensayo 2) encontramos por segundo año consecutivo (Figura 1D) que Basagran (Bentazon) y Herbadox (Phendimetalin) permitieron un control adecuado, pero no superior al de Sencor (Metribuzin). Cention (Diuron), si bien controló efectivamente a la maleza, también afectó al cultivo y su efecto residual fue prolongado. Gesagart (Prometrina), Iloxan (Diclofop metil) y Triflurex (Trifluralina) tampoco constituyeron buenas alternativas para el control de $S$. arvensis. 
No se debe descartar la posibilidad de encontrar resistencia en esta maleza, por la elevada presión de selección que se ocasionaría por el empleo continuado de herbicidas, como lo reportan Bachthaler, et al (1984), en especies de dicotiledóneas en América del Norte y Europa Occidental, principalmente de herbicidas del grupo de las Triazinas como lo es Metribuzin (Sencor). Este hecho pone de manifiesto la gran importancia de contar con otras alternativas de herbicidas como lo son Herbadox (Pendimetalin) y Basagran (Bentazon).

Tabla 4. Análisis marginal de los tratamientos no dominados de los ensayos "Tipos de herbicida" (ensayos 2).

\section{Campaña 1993-94}

\begin{tabular}{|c|c|c|c|c|c|c|}
\hline No. & Tratamiento & $\begin{array}{c}\text { neto } \\
\text { (\$US/ha) }\end{array}$ & $\begin{array}{c}\text { Total } \\
\text { costos } \\
\text { variables } \\
\text { (\$US/ha) }\end{array}$ & $\begin{array}{l}\text { Beneficio } \\
\text { neto } \\
\text { marginal } \\
\text { (\$US/ha) }\end{array}$ & $\begin{array}{c}\text { Costo } \\
\text { variable } \\
\text { marginal } \\
\text { (\$US/ha) }\end{array}$ & $\begin{array}{c}\text { Tasa de } \\
\text { Retorno } \\
\text { Marginal } \\
(\%)\end{array}$ \\
\hline $\begin{array}{l}2 \\
4\end{array}$ & $\begin{array}{l}\text { Sencor } \times \text { Dm } \\
\text { Basagran } \times \text { Dm }\end{array}$ & $\begin{array}{l}3,314 \\
3,075\end{array}$ & $\begin{array}{l}25 \\
22\end{array}$ & $\begin{array}{l}239 \\
103\end{array}$ & $\begin{array}{l}3 \\
6\end{array}$ & $\begin{array}{l}7,967 \\
1,717\end{array}$ \\
\hline $\begin{array}{l}8 \\
6\end{array}$ & $\begin{array}{l}\text { Herbado } \times \text { Dm } \\
\text { Cention } \times \text { Dm }\end{array}$ & $\begin{array}{l}2972 \\
2404\end{array}$ & $\begin{array}{l}16 \\
11\end{array}$ & $\begin{array}{r}568 \\
0\end{array}$ & $\begin{array}{l}5 \\
0\end{array}$ & $\begin{array}{r}11,360 \\
0\end{array}$ \\
\hline
\end{tabular}

Campaña 1994-95

\begin{tabular}{|c|l|l|c|c|c|c|}
\hline No. & Tratamiento & $\begin{array}{c}\text { Beneficio } \\
\text { neto } \\
\text { (\$US/ha) }\end{array}$ & $\begin{array}{c}\text { Total } \\
\text { costos } \\
\text { variables } \\
\text { (\$US/ha) }\end{array}$ & $\begin{array}{c}\text { Beneficio } \\
\text { neto } \\
\text { marginal } \\
\text { (\$US/ha) }\end{array}$ & $\begin{array}{c}\text { Costo } \\
\text { variable } \\
\text { marginal } \\
\text { (\$US/ha) }\end{array}$ & $\begin{array}{c}\text { Tasa de } \\
\text { Retorno } \\
\text { Marginal } \\
\text { (\%) }\end{array}$ \\
\hline 1 & Rencor & 1,504 & 34 & 192 & 5 & 3,840 \\
2 & Basagran & 1,312 & 29 & 114 & 8 & 1,425 \\
3 & Herbados & 1,198 & 21 & 220 & 7 & 3,143 \\
4 & Cention & 978 & 14 & 91 & 3 & 3,033 \\
5 & Gesagart & 887 & 11 & 53 & 2.5 & 2,120 \\
6 & Triflurex & 834 & 8.5 & 0 & 0 & 0 \\
\hline
\end{tabular}

Dm = Dosis mínima .

En la Tabla 4 se muestra el análisis económico de los tratamientos no dominados en los ensayos "Tipos de herbicida" (Ensayos 2) de ambas campañas.

La diferencia en el Costo Variable Total entre los tratamientos, sólo se debió al precio de los herbicidas. Los Beneficios Netos y las altas Tasas de Retorno en ambas campañas, muestran económicamente a los herbicidas Sencor, Basagran y Herbadox como las mejores alternativas, pudiendo aplicarse en sus dosis mínimas. 


\section{Discusión y Conclusiones}

El "aporque" del cultivo de papa es una practica cultural común entre los productores, por los beneficios que conlleva. Los pequeños agricultores de la región andina realizan esta operación en una y hasta en dos oportunidades durante el cultivo, utilizando herramientas manuales. En estas condiciones, esta práctica manual, cuando es ejecutada apropiadamente y en forma oportuna permite entre sus otras ventajas, eliminar las malezas que se desarrollan en los entresurcos y aquellas que se desarrollan sobre los surcos, favoreciendo así a una mejor productividad del cultivo. Este aspecto se confirmó con este estudio, porque el testigo sin aplicación de herbicidas y sólo con el aporque del cultivo, permitió incrementar sus rendimientos.

Cuando el aporque se realiza con tracción animal o mecánica, la eliminación de la maleza no es completa, ya que los implementos sólo logran voltear las malezas que se encuentran en los entresurcos y no así aquellas que se encuentran sobre el surco, al costado de las plantas del cultivo. Estudios realizados por la Universidad Mayor de San Marcos (UMSS) con los proyectos PROMETA y PROMASEL sobre el deshierbe mecánico con tracción animal, utilizando una escardadora que podría ser considerada como un componente complementario para el control de las malezas en campos de agricultores (Crespo 2001). El control químico de malezas en este tipo de agricultura se constituye en una alternativa favorable, siempre y cuando se lo ejecute de una manera apropiada y en forma oportuna. Los resultados de este estudio permiten recomendar para este tipo de agricultura, el uso del herbicida Sencor (Metribizin) aplicado en forma pre-emergente y como posibles alternativas a los herbicidas Basagran (Bentazon) aplicado en forma pos-emergente y Herbadox (Pendimetalin) aplicado en forma pre-emergente. Esta alternativa debe ahora ser validada en campos de agricultores en comparación con el método tradicional y con el deshierbe mecánico mejorado mencionado arriba.

\section{Agradecimientos}

Expresamos nuestros agradecimientos al Ing. Rubén Botello y al Tec. Modesto Totora por la valiosa colaboración prestada durante la ejecución de los experimentos. 


\section{Referencias Bibliográficas}

1. Bachthaler, G.; Kess, H.; Dinzenhofer, B. 1984. Basf, Reportes Agrícolas. El desarrollo de líneas de malas hierbas resistentes tras el empleo continuado de herbicidas, especialmente de triazinas. Boletín de Noticias del Servicio Alemán de Protección de los Vegetales, 35(11 )83. p. 16-22.

2. Crespo, R. 2001. Adaptación y evaluación de escardadoras para tracción animal equina en sistemas agrícolas de ladera. Tesis. Universidad Mayor de San Simón, Facultad de Ciencias Agrícolas y Pecuarias, Cochabamba, Bolivia.

3. García, T.; Quintanilla, F. 1991. Fundamentos Sobre Malas Hierbas y Herbicidas. Edit. Mundi Prensa, Madrid, España. 348 p.

4. Terrazas, X.; Germain, N. 1994. Control de Spergula arvensis L. en el cultivo de trigo. Edit. Revista de agricultura, Universidad Mayor de San Simón, Facultad de Ciencias Agrícolas y Pecuarias, Cochabamba, Bolivia. p. 41-44.

5. Horton, D. 1982. Análisis de presupuestos parciales para la investigación en papa al nivel de finca. Boletín de Información Técnica No. 16. Centro Internacional de la Papa, Lima, Perú. 15 P.

6. Von Hertwing, K. 1983. Manual de herbicidas, desfolhantes, dessecantes, fitorreguladores e bioestimulantes. Edit. Agronómica Ceres, 2 ed, Sao Paulo. 670 p. 